\title{
Epidemiologic Profile of Erectile Dysfunction in Patients with Systemic Lupus Erythematosus: The Latin American Landscape
}

\author{
Javier Merayo-Chalico, Ana Barrera-Vargas, Sandra Morales-Padilla, Roberto Reyna-De la Garza, \\ Ricardo Vázquez-Rodríguez, Jonathan Campos-Guzmán, Jorge Alcocer-Varela, \\ Mariano Sotomayor, Carlos Abud-Mendoza, Marco Martínez-Martínez, Iris Colunga-Pedraza, \\ Christian Uriarte-Hernández, Roberto Acosta-Hernández, Daniel Fajardo, Conrado García-García, \\ Diana Padilla-Ortíz, and Diana Gómez-Martín
}

\begin{abstract}
Objective. The aim of this study was to describe the prevalence of erectile dysfunction (ED), as well as associated demographic and clinical features, in men with systemic lupus erythematosus (SLE), by means of a systematic, standardized evaluation.

Methods. We performed a transversal study in 8 tertiary care centers in Latin America. We included male patients $\geq 16$ years who fulfilled $\geq 4$ American College of Rheumatology criteria for SLE and had regular sexual activity, and evaluated them with the International Index of Erectile Function-5 questionnaire. Relevant demographic, clinical, and serological characteristics were recorded. We included 2 control groups: the first was made up of healthy men and the second of men with autoimmune diseases other than SLE (non-SLE group).

Results. We included 590 subjects (174 SLE, 55 non-SLE, and 361 healthy controls). The prevalence of ED in the SLE group was $69 \%$. Mean age in that group was $36.3 \pm 1.03$ years. Among SLE patients with and without ED, these factors were significantly different: the presence of persistent lymphopenia $(\mathrm{p}=0.006)$, prednisone dose $(9.3 \pm 1.2$ vs $5.3 \pm 1.3 \mathrm{mg}, \mathrm{p}=0.026)$, and the Systemic Lupus International Collaborating Clinics damage score $(1.25 \pm 0.14$ vs $0.8 \pm 0.16$ points, $\mathrm{p}=0.042)$. Independent risk factors for ED in patients with SLE were persistent lymphopenia (OR 2.79, 95\% CI 1.37-5.70, p = 0.001 ) and corticosteroid use in the previous year (OR 2.15, 95\% CI 1.37-3.37, p = 0.001).

Conclusion. Regardless of comorbidities, treatment (excluding steroids), and type of disease activity, patients with SLE have a high prevalence of ED, especially considering that most patients are young. Recent corticosteroid use and persistent lymphopenia, which could be related to endothelial dysfunction, are risk factors for this complication in men with SLE. (First Release January 15 2019; J Rheumatol 2019;46:397-404; doi:10.3899/jrheum.180292)
\end{abstract}

Key Indexing Terms:

SYSTEMIC LUPUS ERYTHEMATOSUS LYMPHOPENIA

\section{ERECTILE DYSFUNCTION CORTICOSTEROIDS}

From the Department of Immunology and Rheumatology, and Department of Urology, Instituto Nacional de Ciencias Médicas y Nutrición Salvador Zubirán, Mexico City; Department of Rheumatology, Hospital Central Dr. Ignacio Morones Prieto, San Luis Potosí, Mexico; Department of Rheumatology, Hospital Universitario Dr. José E. González, Monterrey, Mexico; Department of Internal Medicine, Hospital Metropolitano Vivian Pellas, Managua, Nicaragua; Department of Rheumatology, Instituto Salvadoreño del Seguro Social, San Salvador, El Salvador; Department of Rheumatology, Instituto Mexicano del Seguro Social, Guadalajara,

Mexico; Department of Rheumatology, Hospital General de Mexico Dr. Eduardo Liceaga, Mexico City, Mexico; Department of Internal Medicine, Hospital Universitario de la Samaritana, Bogotá, Colombia.

J. Merayo-Chalico, MD, Department of Immunology and Rheumatology, Instituto Nacional de Ciencias Médicas y Nutrición Salvador Zubirán; A. Barrera-Vargas, MD, Department of Immunology and Rheumatology, Instituto Nacional de Ciencias Médicas y Nutrición Salvador Zubirán; S. Morales-Padilla, MD, Department of Immunology and Rheumatology, Instituto Nacional de Ciencias Médicas y Nutrición Salvador Zubirán; R. Reyna-De la Garza, MD, Department of Immunology and Rheumatology, Instituto Nacional de Ciencias Médicas y Nutrición Salvador Zubirán; R. Vázquez-Rodríguez, MD, Department of

\begin{abstract}
Immunology and Rheumatology, Instituto Nacional de Ciencias Médicas y Nutrición Salvador Zubirán; J. Campos-Guzmán, MD, Department of Immunology and Rheumatology, Instituto Nacional de Ciencias Médicas y Nutrición Salvador Zubirán; J. Alcocer-Varela, PhD, Department of Immunology and Rheumatology, Instituto Nacional de Ciencias Médicas y Nutrición Salvador Zubirán; M. Sotomayor, MD, Department of Urology, Instituto Nacional de Ciencias Médicas y Nutrición Salvador Zubirán; C. Abud-Mendoza, MD, Department of Rheumatology, Hospital Central Dr. Ignacio Morones Prieto; M. Martínez-Martínez, MD, Department of Rheumatology, Hospital Central Dr. Ignacio Morones Prieto; I. Colunga-Pedroza, MD, Department of Rheumatology, Hospital Universitario; C. Uriarte-Hernández, MD, Department of Internal Medicine, Hospital Metropolitano Vivian Pellas; R. Acosta-Hernández, $M D$, Department of Rheumatology, Instituto Salvadoreño del Seguro Social; D. Fajardo, MD, Department of Rheumatology, Instituto Mexicano del Seguro Social; C. García-García, MD, Department of Rheumatology, Hospital General de Mexico; D. Padilla-Ortiz, MD, Department of Internal Medicine, Hospital Universitario de la Samaritana; D. Gómez-Martín, PhD, Department of Immunology and Rheumatology, Instituto Nacional de Ciencias Médicas y Nutrición Salvador Zubirán. Address correspondence to D. Gómez-Martín, Department of Immunology
\end{abstract}

Personal non-commercial use only. The Journal of Rheumatology Copyright @ 2019 . All rights reserved. 
and Rheumatology, Instituto Nacional de Ciencias Médicas y Nutrición Salvador Zubirán, Vasco de Quiroga 15, Tlalpan 14080, Mexico City, Mexico.E-mail: diana.gomezm@incmnsz.mx

Accepted for publication September 11, 2018.

Systemic lupus erythematosus (SLE) predominantly affects women ${ }^{1}$, but usually has worse prognosis, higher activity scores, and an increased mortality in men ${ }^{2,3,4}$, both globally as well as in Latin American patients ${ }^{5}$. Sexual function, which directly affects quality of life, has different domains that can be affected by disease. In male patients, erectile function is especially relevant, because this disorder is associated with both neuropsychiatric problems (mainly anxiety and depression) and cardiovascular (CV) disease ${ }^{6}$. Whereas there are different studies regarding sexual function in men with autoimmune diseases [mainly rheumatoid arthritis (RA), spondyloarthritis ( $\mathrm{SpA}$ ), and systemic sclerosis $(\mathrm{SSc})]^{7,8,9,10,11,12,13}$, as well as studies about reproductive function ${ }^{14,15,16,17,18}$ and alterations regarding penile anthropometry in SLE ${ }^{19}$, information about erectile dysfunction (ED) in patients with SLE is quite scant $20,21,22$.

Males with SLE are mostly young. However, many of them are taking intense immunosuppressive therapies or high-dose steroids, and are at risk for accelerated atherosclerosis ${ }^{2,3,4}$. Taking these factors into account, as well as the absence of studies performed with a validated instrument that allows a systematic analysis about ED in this group of patients, the main aim of our study was to describe the prevalence, risk factors, and features associated with ED in patients with SLE.

\section{MATERIALS AND METHODS}

We performed a transversal study in 8 tertiary care centers throughout Latin America (5 in Mexico, 1 Nicaragua, 1 in El Salvador, and 1 in Colombia) between October 2015 and November 2016. All subjects were included during outpatient visits. Three study groups were formed: men with SLE, men with autoimmune diseases other than SLE (non-SLE group), and healthy controls. In the first group, patients were $\geq 16$ years old, fulfilled $\geq$ 4 American College of Rheumatology classification criteria, and had regular sexual activity in the prior 6 months (with intercourse at least once per week). Patients with other autoimmune diseases were excluded [except for antiphospholipid syndrome (APS), chronic viral infections (human immunodeficiency virus, hepatitis B or C), cancer, and late-onset SLE (diagnosis after the age of $50 \mathrm{yrs}]^{23}$. We included 2 control groups -1 with autoimmune diseases other than SLE (non-SLE group, which included RA, SpA, SSc, Sjögren syndrome, systemic vasculitides, adult-onset Still disease, inflammatory myopathies, and primary APS), and the other with healthy controls from each center, who were matched by age with the SLE subjects. Those with diagnosis of a chronic disease or who were using any prescription drug were excluded from this last group. In all groups, patients with incomplete clinical information in their clinical records were excluded.

All included subjects filled out the 5-item version of the International Index of Erectile Function (IIEF-5; Supplementary Table 1, available from the authors on request) in the Spanish-validated version ${ }^{24,25}$. The questionnaire was completed anonymously by each subject, in an isolated room, without any other person present, and was finally placed into a covered box. In the IIEF-5, the lowest score per question is 1 and the highest is 5. A normal erectile function is considered when scores are between 22 and 25, and ED is graded as mild (17-21 points), mild to moderate (12-16 points), moderate (8-11 points), and severe (5-7 points) ${ }^{24}$. Two last questions were added for patients, asking whether their rheumatologist had queried them about sexual issues during the previous 3 visits, and whether they would like their rheumatologist to inquire about those issues.

For patients in the SLE group, we recorded demographic information, history of disease activity, comorbidities, autoantibody profile, the SLE Disease Activity Index (SLEDAI ${ }^{26}$ activity score, the Systemic Lupus International Collaborating Clinics (SLICC) Damage Index ${ }^{27}$, and immunosuppressive and nonimmunosuppressive medications. Active disease was defined as SLEDAI $\geq 6$ points. Lymphopenia was defined as a total lymphocyte count $\leq 1000$ cells $/ \mathrm{m}^{3}$ and persistent lymphopenia as at least 3 consecutive measurements $\leq 1000$ cells $/ \mathrm{m}^{3}$. For patients in the non-SLE group, disease activity was established according to the physician's global assessment. Briefly, we defined disease activity in this group of patients when it was considered after the clinical evaluation by their attending rheumatologist, and immunosuppressive treatment was adjusted accordingly. Regarding healthy controls, age and anthropometric measurements were recorded.

The study was approved by the local Research and Ethics Institutional Committee (Ref 1661). Variables were described in terms of mean and SD or proportions, as convenient. For comparison between groups, the SLE group was compared with each control group; chi-square test was used for categorical variables or Student $t$ test for quantitative variables. Association between variables was assessed by OR with $95 \%$ CI. Variables that showed statistical significance in the univariate analysis or those with clinical relevance were included in the multivariate analysis, which was performed through binary logistic regression. A p value $<0.05$ was considered statistically significant. Statistical analyses were performed with support of the software SPSS, version 21

\section{RESULTS}

We included 590 subjects (SLE $\mathrm{n}=174$, non-SLE $\mathrm{n}=55$, healthy controls $n=361$ ). According to the setting, the patients with SLE were included as follows: Instituto Nacional de Ciencias Médicas y Nutrición Salvador Zubirán (Mexico City, Mexico), 43 patients; Hospital Central Ignacio Morones Prieto (San Luis Potosí, Mexico), 24 patients; Hospital Universitario Dr. José E. González (Monterrey, Mexico), 22 patients; Hospital Metropolitano Vivian Pellas (Managua, Nicaragua), 19 patients; Instituto Salvadoreño del Seguro Social (San Salvador, El Salvador), 18 patients; Instituto Mexicano del Seguro Social (Guadalajara, Mexico), 18 patients; Hospital General de México Dr. Eduardo Liceaga (Mexico City, Mexico), 15 patients; Hospital Universitario de la Samaritana (Bogotá, Colombia), 15 patients. Patients in the non-SLE group had the following diagnoses: RA (34.54\%), SpA (20\%), primary APS (14.54\%), inflammatory myopathies (7.27\%), SSc (5.45\%), systemic vasculitides (9.09\%), juvenile idiopathic arthritis (3.63\%), Sjögren syndrome (1.81\%), relapsing polychondritis (1.81\%), and Still disease (1.81\%). Table 1 displays the main clinical and demographic features in the SLE and non-SLE groups. Information regarding healthy controls is shown in Supplementary Table 2 (available from the authors on request).

The prevalence of ED in the SLE group was $68.96 \%$ versus $23.26 \%$ in healthy controls $(\mathrm{p}=0.001)$. There was no statistically significant difference in the ED prevalence when compared with the non-SLE group, but patients with SLE presented with $\mathrm{ED}$ at a significantly younger age $(36.3 \pm 1.03$ in SLE vs $46.3 \pm 2.2$ yrs in non-SLE, $\mathrm{p}<0.0001$; Table 1 ). 
Table 1. Demographic, clinical and serological features of SLE and non-SLE patients with and without erectile dysfunction.

\begin{tabular}{|c|c|c|c|c|c|c|}
\hline \multirow[t]{2}{*}{ Variables } & \multicolumn{3}{|c|}{ With Erectile Dysfunction, $n=153$} & \multicolumn{3}{|c|}{ Without Erectile Dysfunction, $\mathrm{n}=76$} \\
\hline & $\begin{array}{l}\text { SLE, } \mathrm{n}=120 \\
\text { mean } \pm \text { SEM }\end{array}$ & $\begin{array}{c}\text { Non-SLE, } \mathrm{n}=33 \\
\text { mean } \pm \text { SEM }\end{array}$ & $\mathrm{p}$ & $\begin{array}{l}\text { SLE, } \mathrm{n}=54 \\
\text { mean } \pm \text { SEM }\end{array}$ & $\begin{array}{c}\text { Non-SLE, } \mathrm{n}=22 \\
\text { mean } \pm \text { SEM }\end{array}$ & $\mathrm{p}$ \\
\hline \multicolumn{7}{|l|}{ Demographic } \\
\hline Age, yrs & $36.3 \pm 1.03$ & $46.3 \pm 2.2$ & $<0.0001$ & $32.5 \pm 1.27$ & $40.5 \pm 2.25$ & 0.002 \\
\hline Weight, kg & $77.7 \pm 1.41$ & $77.5 \pm 2.8$ & 0.92 & $77 \pm 1.83$ & $77.6 \pm 3.77$ & 0.87 \\
\hline Height, cm & $171 \pm 1.4$ & $166 \pm 5.4$ & 0.18 & $171 \pm 0.99$ & $168 \pm 1.67$ & 0.09 \\
\hline $\mathrm{BMI}, \mathrm{kg} / \mathrm{m}^{2}$ & $26.9 \pm 0.46$ & $26.2 \pm 0.68$ & 0.44 & $26 \pm 0.60$ & $27.1 \pm 1.03$ & 0.37 \\
\hline Time since diagnosis, yrs & $8.1 \pm 0.68$ & $10.5 \pm 1.32$ & 0.10 & $7.9 \pm 1.03$ & $10.6 \pm 1.69$ & 0.16 \\
\hline Activity of connective tissue disease $\mathrm{a}^{\mathrm{a}}$ & $69(57)$ & $7 / 33(21)$ & $<0.0001$ & $28 / 54(51)$ & $4 / 22(18)$ & 0.01 \\
\hline \multicolumn{7}{|l|}{ Laboratory features } \\
\hline Hemoglobin, g/dl & $14.1 \pm 0.24$ & $15.2 \pm 0.33$ & 0.02 & $16.2 \pm 1.18$ & $15.4 \pm 0.54$ & 0.66 \\
\hline Leukocytes, cells $/ \mu \mathrm{l} \times 10^{3}$ & $6.3 \pm 0.22$ & $7.7 \pm 0.43$ & 0.006 & $10.4 \pm 3.23$ & $10.4 \pm 3.02$ & 0.99 \\
\hline Lymphocytes, cells/ $\mu \mathrm{l}$ & $1333 \pm 54$ & $1707 \pm 104$ & 0.002 & $1644 \pm 106$ & $1774 \pm 190$ & 0.53 \\
\hline Platelets, cells $/ \mu 1 \times 10^{3}$ & $232 \pm 7.8$ & $271 \pm 20$ & 0.03 & $229 \pm 11.3$ & $278 \pm 22.1$ & 0.03 \\
\hline Creatinine, $\mathrm{mg} / \mathrm{dl}$ & $1.72 \pm 0.31$ & $0.87 \pm 0.04$ & 0.008 & $1.38 \pm 0.23$ & $1.01 \pm 0.18$ & 0.35 \\
\hline APS serology, n $(\%)$ & $29(24)$ & $5(15)$ & 0.47 & $13(24)$ & $4(18)$ & 0.76 \\
\hline Use of immunosuppressive treatment, $\mathrm{n}(\%)$ & $108(90)$ & $26(78)$ & 0.13 & $46(85)$ & $17(77)$ & 0.31 \\
\hline Prednisone, $\mathrm{n}(\%)$ & $82(68)$ & $14(42)$ & 0.008 & $28(51)$ & $8(36)$ & 0.31 \\
\hline Current dose, mg/day & $9.3 \pm 1.20$ & $3.5 \pm 1.12$ & 0.017 & $5.32 \pm 1.29$ & $4.88 \pm 1.96$ & 0.85 \\
\hline Cumulative dose, previous year, $\mathrm{mg}$ & $2525 \pm 337$ & $1555 \pm 590$ & 0.15 & $2398 \pm 591$ & $1236 \pm 491$ & 0.18 \\
\hline Cumulative dose, 5 yrs, $\mathrm{mg}$ & $11584 \pm 1167$ & $5016 \pm 1395$ & 0.003 & $11278 \pm 1836$ & $5835 \pm 2328$ & 0.09 \\
\hline Non-exposure to any steroid in previous year, $\mathrm{n}(\%)$ & $29(24)$ & $15(45)$ & 0.029 & $23(42)$ & $12(54)$ & 0.45 \\
\hline Azathioprine, n (\%) & $39(32)$ & $6(18)$ & 0.13 & $18(33)$ & $2(9)$ & 0.04 \\
\hline Current dose, mg/day & $32.9 \pm 4.7$ & $17.4 \pm 6.5$ & 0.11 & $31.9 \pm 7.22$ & $10.2 \pm 5.85$ & 0.02 \\
\hline Antimalarial, $\mathrm{n}(\%)$ & $73(60)$ & $8(24)$ & $<0.0001$ & $34(62)$ & $4(18)$ & 0.01 \\
\hline Current dose, mg/day & $137.2 \pm 11.8$ & $37.8 \pm 14.1$ & $<0.0001$ & $123.1 \pm 15.1$ & $31.8 \pm 14.9$ & $<0.0001$ \\
\hline Methotrexate, $\mathrm{n}(\%)$ & $16(13)$ & $15(45)$ & $<0.0001$ & $7(12)$ & $10(45)$ & 0.005 \\
\hline Current dose, $\mathrm{mg} /$ week & $2.21 \pm 0.53$ & $7.73 \pm 1.58$ & $<0.0001$ & $1.60 \pm 0.71$ & $7.73 \pm 2.10$ & 0.01 \\
\hline Mycophenolate mofetil, n (\%) & $48(40)$ & $1(3)$ & $<0.0001$ & $18(33)$ & $1(4)$ & 0.008 \\
\hline Current dose, mg/day & $665 \pm 88$ & $37 \pm 37.8$ & $<0.0001$ & $517 \pm 123$ & $90.9 \pm 90.9$ & 0.07 \\
\hline Cumulative dose, 5 yrs, g & $804 \pm 154.4$ & $195 \pm 195$ & 0.034 & $743 \pm 179$ & $235 \pm 235$ & 0.09 \\
\hline Cyclophosphamide exposure previous 6 mos, $\mathrm{n}(\%)$ & $12(10)$ & $1(3)$ & 0.30 & $4(7)$ & $0(0)$ & 0.31 \\
\hline Cumulative dose, $6 \mathrm{mos}, \mathrm{g}$ & $0.36 \pm 0.13$ & $0.18 \pm 0.18$ & 0.52 & $0.31 \pm 0.18$ & $0 \pm 0$ & 0.09 \\
\hline Cyclophosphamide exposure, lifelong, n (\%) & $62(51)$ & $3(9)$ & $<0.0001$ & $23(42)$ & $1(4)$ & 0.001 \\
\hline Cumulative dose, lifelong, $g$ & $10.9 \pm 3.6$ & $0.22 \pm 0.19$ & 0.004 & $11.5 \pm 6.2$ & $0.70 \pm 0.52$ & 0.09 \\
\hline Nonimmunosuppressive treatment, $\mathrm{n}(\%)$ & $97(80)$ & $25(75)$ & 0.65 & $41(75)$ & $15(68)$ & 0.56 \\
\hline Any comorbidities, $\mathrm{n}(\%)$ & $63(52)$ & $14(42)$ & 0.33 & $24(44)$ & $10(45)$ & 1 \\
\hline Type 2 diabetes mellitus, $\mathrm{n}(\%)$ & $6(5)$ & $6(18)$ & 0.02 & $0(0)$ & $4(18)$ & 0.006 \\
\hline Hypertension, $\mathrm{n}(\%)$ & $44(36)$ & $14(42)$ & 0.54 & $14(25)$ & $4(18)$ & 0.56 \\
\hline Major depressive disorder, $\mathrm{n}(\%)$ & $5(4)$ & $0(0)$ & 0.32 & $3(5)$ & $0(0)$ & 0.54 \\
\hline Dyslipidemia $^{\mathrm{b}}, \mathrm{n}(\%)$ & $33(27)$ & $9(27)$ & 1 & $12(22)$ & $6(27)$ & 0.76 \\
\hline Coronary heart disease ${ }^{\mathrm{c}}, \mathrm{n}(\%)$ & $5(4)$ & $0(0)$ & 0.58 & $0(0)$ & $2(9)$ & 0.08 \\
\hline Arterial and/or venous thrombosis, $\mathrm{n}(\%)$ & $32(26)$ & $5(15)$ & 0.25 & $12(22)$ & $2(9)$ & 0.21 \\
\hline Peripheral arterial disease $^{\mathrm{d}}, \mathrm{n}(\%)$ & $0(0)$ & $1(3)$ & 0.21 & $0(0)$ & $0(0)$ & ND \\
\hline Smoking $^{\mathrm{e}}, \mathrm{n}(\%)$ & $32(26)$ & $7(21)$ & 0.90 & $11(20)$ & $4(18)$ & 1 \\
\hline Genitourinary surgery ${ }^{\mathrm{f}}, \mathrm{n}(\%)$ & $6(5)$ & $2(6)$ & 0.68 & $3(5)$ & $0(0)$ & 0.55 \\
\hline
\end{tabular}

Values shown in bold represent statistically significant $\mathrm{p}$ values. ${ }^{\mathrm{a}}$ Disease activity was defined for non-SLE as an increase or addition of any immunosuppressive treatment according to physician's assessment; activity for SLE group was defined by SLEDAI $\geq 6$ points. ${ }^{b}$ Hypercholesterolemia $\geq 200 \mathrm{mg} / \mathrm{dl}(5.18 \mathrm{mmol} / \mathrm{l})$ and/or hypertriglyceridemia $\geq 150 \mathrm{mg} / \mathrm{dl}(1.69 \mathrm{mmol} / \mathrm{l}) .{ }^{\mathrm{c}}$ Proven by angiography in the last 10 years. ${ }^{\mathrm{d}}$ Proven by angiography and/or Doppler ultrasound in the last 10 years. ${ }^{\mathrm{e}}$ Current or in the past 5 years. ${ }^{\mathrm{f}}$ Any urinary tract, prostate, penis, or testicle surgery, excluding circumcision. SLE: systemic lupus erythematosus; SEM: standard error of the mean; BMI: body mass index; APS: antiphospholipid syndrome; SLEDAI: SLE Disease Activity Index; ND: not determined.

ED in the SLE group was mostly mild to moderate (108/120 patients, 90\%). Men with SLE had a mean IIEF-5 score of $17.2 \pm 0.33$ points. Table 2 shows individual scores per question in patients with SLE, as well as total score and the ED category. Table 1 shows the comparison between patients with and without ED in the SLE and non-SLE groups.
Remarkably, prednisone use was more frequent in patients with ED than in those without it $(\mathrm{p}=0.026)$. Regarding comorbidities, the only difference among groups was a higher prevalence of type 2 diabetes mellitus (DM) in non-SLE patients, but there was no difference in patients with or without ED.

$$
\text { Personal non-commercial use only. The Journal of Rheumatology Copyright } \subset \text { 2019. All rights reserved. }
$$


Table 2. Itemized International Index of Erectile Function-5 in patients with SLE.

\begin{tabular}{lccc}
\hline Variable & SLE with ED, $\mathrm{n}=120$ & SLE without ED, $\mathrm{n}=54$ & $\mathrm{p}$ \\
\hline Question 1 (1-5 points) & $3.03 \pm 0.08$ & $4.17 \pm 0.09$ & $<\mathbf{0 . 0 0 0 1}$ \\
Question 2 (1-5 points) & $3.41 \pm 0.10$ & $4.89 \pm 0.04$ & $\mathbf{0 . 0 0 0 1}$ \\
Question 3 (1-5 points) & $3.32 \pm 0.08$ & $4.81 \pm 0.05$ & $\mathbf{< 0 . 0 0 0 1}$ \\
Question 4 (1-5 points) & $3.84 \pm 0.08$ & $4.89 \pm 0.04$ & $\mathbf{0 . 0 0 0 1}$ \\
Question 5 (1-5 points) & $3.63 \pm 0.09$ & $4.89 \pm 0.04$ & $\mathbf{< . 0 0 0 1}$ \\
Total score (5-25 points) & $17.2 \pm 0.33$ & $23.6 \pm 0.14$ & $\mathbf{0 . 0 0 0 1}$ \\
Erectile dysfunction category, n (\%) & & & \\
$\quad$ Mild & $84 / 120(70)$ & NA & \\
$\quad$ Mild to moderate & $24 / 120(20)$ & NA & \\
$\quad$ Moderate & $9 / 120(7.5)$ & NA & \\
Severe & $3 / 120(2.5)$ & NA & \\
\hline
\end{tabular}

Question 1-5 and total scores are mean \pm SEM. Values shown in bold represent statistically significant $\mathrm{p}$ values. SLE: systemic lupus erythematosus; ED: erectile dysfunction; NA: not applicable.

Table 3 contains relevant information about all patients with SLE. As shown, SLE patients with ED were slightly older than those without ED $(36.3 \pm 1.03$ vs $32.5 \pm 1.27 \mathrm{yrs}$, $\mathrm{p}=0.022$ ), without differences in weight, height, body mass index, or time since SLE diagnosis. SLE patients with ED had a lower count of total lymphocytes at the time of the study $(p=0.005)$, as well as a higher prevalence of persistent lymphopenia $(\mathrm{p}=0.006)$. Regarding immunosuppressive therapy, the use of any of these drugs was equal between groups $(\mathrm{p}=0.6)$. However, patients with ED had a higher frequency of exposure to any dose of corticosteroids in the previous year $(\mathrm{p}=0.019)$, and also a higher prednisone dose at the time of the study $(9.31 \pm 1.20$ vs $5.32 \pm 1.29 \mathrm{mg} /$ day, $\mathrm{p}=0.02)$. There were no other significant differences regarding the rest of the immunosuppressive treatment between groups, both at the time of the study and with cumulative doses. There were no relevant differences in comorbidities $(p=0.41)$ or in nonimmunosuppressive treatments $(\mathrm{p}=0.54)$ between groups. Further, there was no difference regarding disease activity (SLEDAI score $4.89 \pm$ 0.54 vs $3.65 \pm 0.52, \mathrm{p}=0.16$ ), but patients with ED had significantly more damage accrual according to the SLICC Damage Index ( $1.25 \pm 0.14$ vs $0.80 \pm 0.16$ points, $p=0.042$ )

After multivariate analysis, risk factors associated with ED in patients with SLE were persistent lymphopenia (OR $2.79,95 \%$ CI $1.37-5.70, \mathrm{p}=0.001)$ and exposure to any corticosteroid dose in the previous year (OR 2.15, 95\% CI 1.37-3.37, $\mathrm{p}=0.001$ ).

Finally, we found that most patients who attended the different rheumatology clinics were not questioned about erectile function (86\%), at least during the previous 3 visits. Also, most patients $(82 \%)$ would consider it appropriate to discuss erectile and sexual function in their usual visits (Figure 1).

\section{DISCUSSION}

To our knowledge, this is the first study to evaluate the prevalence of ED in patients with SLE by using an adequate, validated questionnaire ${ }^{24}$. We found a prevalence close to $70 \%$, whereas in the healthy population the prevalence of ED is between 10 and $22 \%{ }^{28}$. Our findings are similar to those described in patients with DM; however, it is important to note that patients with type 2 diabetes in whom that prevalence has been found were 22 years older than our population, on average ${ }^{29}$. There are various possible explanations for such a high prevalence of ED in young patients with SLE (mean age $36.3 \mathrm{yrs}$ ). Currently, well-known risk factors for ED are obesity (RR 1.9, 95\% CI 1.6-2.2), smoking (RR 1.5, 95\% CI $1.3-1.7)^{30}$, DM (OR 3, 95\% CI 1.5-5.8), hypertension (OR 2.05, 95\% CI 1.6-2.6), hyperlipidemia (OR 2.2, 95\% CI 1.4-3.7), lower urinary tract symptoms (OR 2.2,95\% CI 1.7-2.7), psychological stress (OR 1.6, 95\% CI 1.4-1.9), low physical activity (OR 1.3, 95\% CI 1.1-1.6), and age 31 . Whereas the physiopathology of ED involves multiple mechanisms, including the use of certain drugs, history of pelvic surgery or pelvic irradiation, as well as endocrine, neurologic, and psychogenic factors, the main cause associated with ED is local vascular damage ${ }^{32}$. Interestingly, most of these conventional risk factors were absent in our patients, but the recent use of glucocorticoids was found to be of particular relevance.

Because men with SLE have higher disease activity scores $^{2}$, they use glucocorticoids more frequently and in higher doses ${ }^{33}$. We found glucocorticoid use to be a risk factor for ED. Although the use of systemic corticosteroids has not been previously directly related to $\mathrm{ED}^{34}$, up to $70 \%$ of patients with Cushing syndrome have sexual dysfunction. It has been suggested that cortisol plays an inhibitory role in male sexual response $\mathrm{e}^{35}$. Indeed, hypercortisolism leads to a decreased concentration of luteinizing hormone and testosterone. It also inhibits the pituitary secretion of gonadotrophins and the androgen production in Leydig cells ${ }^{36,37}$. Also, this effect seems to be reversible, which would explain why recent exposure to glucocorticoids was found to be a risk factor for ED, but the cumulative corticosteroid dose was not. Further, glucocorticoids have been associated with

Personal non-commercial use only. The Journal of Rheumatology Copyright @ 2019 . All rights reserved. 
Table 3. Demographic, clinical, and serological features of SLE patients with and without erectile dysfunction.

\begin{tabular}{|c|c|c|c|}
\hline Variable & $\begin{array}{c}\text { SLE without ED, } \\
n=54 \\
\text { mean } \pm \text { SEM }\end{array}$ & $\begin{array}{l}\text { SLE with ED, } \\
\mathrm{n}=120 \\
\text { mean } \pm \text { SEM }\end{array}$ & $\mathrm{p}$ \\
\hline \multicolumn{4}{|l|}{ Demographic } \\
\hline Total IIEF-5 score, points & $23.6 \pm 0.14$ & $17.3 \pm 0.33$ & $<0.0001$ \\
\hline Age, yrs & $32.5 \pm 1.27$ & $36.3 \pm 1.03$ & 0.022 \\
\hline Weight, $\mathrm{kg}$ & $77 \pm 1.83$ & $77.7 \pm 1.41$ & 0.76 \\
\hline Height, cm & $171.9 \pm 0.99$ & $171.4 \pm 1.42$ & 0.83 \\
\hline $\mathrm{BMI}, \mathrm{kg} / \mathrm{m}^{2}$ & $26 \pm 0.60$ & $26.9 \pm 0.46$ & 0.25 \\
\hline Time since diagnosis, yrs & $7.93 \pm 1.03$ & $8.13 \pm 0.68$ & 0.87 \\
\hline SLEDAI score, points & $3.62 \pm 0.52$ & $4.89 \pm 0.54$ & 0.16 \\
\hline SLICC score, points & $0.80 \pm 0.16$ & $1.25 \pm 0.14$ & 0.042 \\
\hline \multicolumn{4}{|l|}{ Laboratory features } \\
\hline Hemoglobin, g/dl & $16.2 \pm 1.18$ & $14.1 \pm 0.24$ & 0.015 \\
\hline Leukocytes, cells $/ \mu \mathrm{l} \times 10^{3}$ & $10.4 \pm 3.23$ & $6.36 \pm 0.22$ & 0.21 \\
\hline Lymphocytes, cells/ $\mu \mathrm{l}$ & $1644 \pm 116$ & $1333 \pm 54.9$ & 0.005 \\
\hline Platelets, cells $/ \mu \mathrm{l} \times 10^{3}$ & $229 \pm 11.3$ & $232 \pm 7.86$ & 0.83 \\
\hline Creatinine, $\mathrm{mg} / \mathrm{dl}$ & $1.38 \pm 0.23$ & $1.72 \pm 0.31$ & 0.48 \\
\hline C3 levels, g/l & $93 \pm 4.59$ & $92.8 \pm 4.28$ & 0.97 \\
\hline C4 levels, g/1 & $18 \pm 1.94$ & $19.8 \pm 1.20$ & 0.38 \\
\hline Anti-dsDNA, IU/ml & $114 \pm 37.3$ & $135 \pm 50.1$ & 0.78 \\
\hline Use of immunosuppressive treatment, $\mathrm{n}(\%)$ & $46(85)$ & $108(90)$ & 0.60 \\
\hline Prednisone, $\mathrm{n}(\%)$ & $28(51)$ & $82(68)$ & 0.043 \\
\hline Current dose, mg/day & $5.32 \pm 1.29$ & $9.31 \pm 1.20$ & 0.02 \\
\hline Cumulative dose, previous year, $\mathrm{mg}$ & $2398 \pm 591$ & $2525 \pm 337$ & 0.85 \\
\hline Cumulative dose, 5 years, $\mathrm{mg}$ & $11278 \pm 1836$ & $11584 \pm 1167$ & 0.88 \\
\hline \multicolumn{4}{|l|}{ Nonexposure to any steroid in previous } \\
\hline year, $\mathrm{n}(\%)$ & $23(42)$ & $29(24)$ & 0.019 \\
\hline Azathioprine & $18(33)$ & $39(32)$ & 1 \\
\hline Current dose, mg/day & $31.9 \pm 7.22$ & $32.9 \pm 4.78$ & 0.91 \\
\hline Antimalarial & $34(62)$ & $73(60)$ & 0.86 \\
\hline Current dose, mg/day & $123 \pm 15$ & $137 \pm 11$ & 0.48 \\
\hline Methotrexate & $7(12)$ & $16(13)$ & 1 \\
\hline Current dose, mg/week & $1.60 \pm 0.71$ & $2.21 \pm 0.53$ & 0.52 \\
\hline Mycophenolate mofetil, n (\%) & $18(33)$ & $48(21)$ & 0.50 \\
\hline Current dose, mg/day & $517 \pm 123$ & $665 \pm 88$ & 0.34 \\
\hline Cumulative dose, 5 yrs, $\mathrm{g}$ & $743 \pm 179$ & $804 \pm 154$ & 0.81 \\
\hline CYC exposure previous 6 mos, $\mathrm{n}(\%)$ & $4(7)$ & $12(10)$ & 0.77 \\
\hline Cumulative dose, 6 mos, $g$ & $0.31 \pm 0.18$ & $0.36 \pm 0.13$ & 0.85 \\
\hline $\mathrm{CYC}$ exposure to any dose, lifelong, n (\%) & $23(42)$ & $62(51)$ & 0.32 \\
\hline Cumulative dose, lifelong, $g$ & $11.5 \pm 6.20$ & $10.9 \pm 3.60$ & 0.92 \\
\hline Nonimmunosuppressive treatment, $\mathrm{n}(\%)$ & $41(75)$ & $97(80)$ & 0.54 \\
\hline Antihypertensive & $26(48)$ & $77(64)$ & 0.06 \\
\hline Antidepressant & $3(5)$ & $6(5)$ & 1 \\
\hline Antidiabetic & $2(3)$ & $5(4)$ & 1 \\
\hline Hypolipidemic & $11(20)$ & $39(32)$ & 0.14 \\
\hline NSAID & $15(27)$ & $36(30)$ & 0.85 \\
\hline Anticoagulation & $8(14)$ & $29(24)$ & 0.22 \\
\hline Any comorbidities, $\mathrm{n}(\%)$ & $24(44)$ & $63(52)$ & 0.41 \\
\hline Type 2 diabetes mellitus & $0(0)$ & $6(5)$ & 0.17 \\
\hline Hypertension & $14(25)$ & $44(36)$ & 0.22 \\
\hline Renal replacement therapy & $2(3)$ & $8(6)$ & 0.72 \\
\hline Postrenal transplantation & $2(3)$ & $5(4)$ & 1 \\
\hline Major depressive disorder & $3(5)$ & $5(4)$ & 0.69 \\
\hline Dyslipidemia $^{a}$ & $12(22)$ & $33(27)$ & 0.57 \\
\hline Coronary heart disease $\mathrm{e}^{\mathrm{b}}$ & $0(0)$ & $5(4)$ & 0.32 \\
\hline Arterial and/or venous thrombosis & $12(22)$ & $32(26)$ & 0.70 \\
\hline Peripheral arterial disease $\mathrm{c}^{\mathrm{c}}$ & $0(0)$ & $0(0)$ & ND \\
\hline Smoking ${ }^{\mathrm{d}}$ & $11(20)$ & $32(26)$ & 0.90 \\
\hline Genitourinary surgery $\mathrm{e}$ & $3(5)$ & $6(5)$ & 1 \\
\hline
\end{tabular}


Table 3. Continued.

\begin{tabular}{lccc}
\hline Variable & $\begin{array}{c}\text { SLE without ED, } \\
\mathrm{n}=54, \\
\text { mean } \pm \text { SEM }\end{array}$ & $\begin{array}{c}\text { SLE with ED, } \\
\mathrm{n}=120, \\
\text { mean } \pm \text { SEM }\end{array}$ & $\mathrm{p}$ \\
\hline SLE (history), $\mathrm{n}(\%)$ & & & \\
Hematological activity & $14(25)$ & $33(27)$ & 1 \\
Persistent lymphopenia & $11(20)$ & $51(42)$ & $\mathbf{0 . 0 0 6}$ \\
Neurological activity & $4(7)$ & $15(12)$ & 0.43 \\
Diffuse alveolar hemorrhage & $2(3)$ & $7(5)$ & 0.72 \\
Renal activity & $23(42)$ & $68(56)$ & 0.13 \\
APS serology & $13(24)$ & $29(24)$ & 1 \\
\hline
\end{tabular}

Values in bold face represent statistically significant $\mathrm{p}$ values. ${ }^{\mathrm{a}}$ Hypercholesterolemia $\geq 200 \mathrm{mg} / \mathrm{dl}$ (5.18 mmol/l) and/or hypertriglyceridemia $\geq 150 \mathrm{mg} / \mathrm{dl}(1.69 \mathrm{mmol} / \mathrm{l}) .{ }^{\mathrm{b}}$ Proven by angiography in the last 10 years. ${ }^{\mathrm{c}}$ Proven by angiography and/or Doppler ultrasound in the last 10 years. ${ }^{\mathrm{d}}$ Current or in the past 5 years. ${ }^{\mathrm{e}}$ Any urinary tract, prostate, penis, or testicle surgery, excluding circumcision. SLE: systemic lupus erythematosus; ED: erectile dysfunction; SEM: standard error of the mean; IIEF: Itemized International Index of Erectile Function; BMI: body mass index; SLEDAI: SLE Disease Activity Index; SLICC: Systemic Lupus International Collaborating Clinics; CYC: cyclophosphamide; NSAID: nonsteroidal antiinflammatory drugs; APS: antiphospholipid síndrome; ND: not determined.

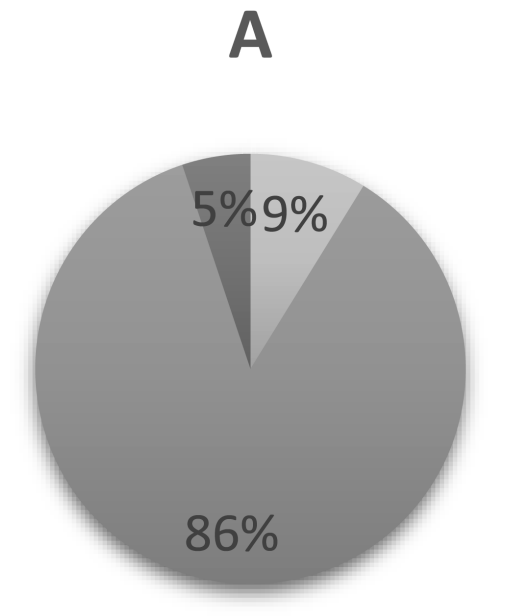

\section{Yes No Don't know}

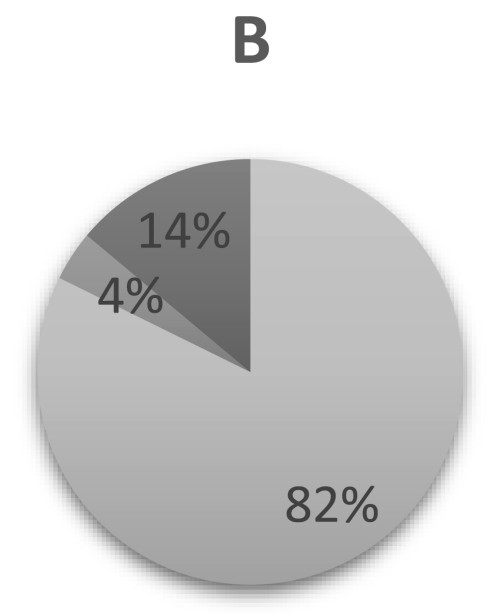

Yes $\quad$ No $\quad$ Don't know

Figure 1. A. The distribution of responses of patients $(\mathrm{n}=174)$ with SLE to this query: Have you been asked about your erectile function in your last 3 visits to the rheumatologist? B. The percentage of responses from patients with SLE regarding their likelihood of being questioned about their erectile function by their rheumatologists (i.e., would you like your rheumatologist to inquire about your erectile function during your regular visits?). SLE: systemic lupus erythematosus.

subclinical atherosclerosis in patients with $\mathrm{SLE}^{38}$, and they are considered an additional risk factor for CV disease ${ }^{39}$. Along with their hormonal actions, their vascular effects could play an additional role in the development of ED in young patients with SLE. ED has been considered as an independent risk factor for major $\mathrm{CV}$ events in the general population ${ }^{40}$. Therefore, it will be relevant to identify SLE patients with ED, not only to offer adequate and timely treatment, but also to assess the presence of other $\mathrm{CV}$ risk factors and to begin an appropriate prevention strategy. Further, ED has been found to directly influence quality of life ${ }^{41}$, which in the case of patients with SLE is already affected by the disease itself ${ }^{42}$.

While it is widely known that cyclophosphamide may cause oligo/azoospermia, infertility, and alterations in the sex hormone profile in men with SLE ${ }^{15,17}$, we did not find recent exposure or cumulative doses to be associated with ED.

Further, we also found persistent lymphopenia to be a risk factor for ED in these patients. This could also be related to vascular damage, and specifically to endothelial dysfunction. Low lymphocyte counts have been associated with accelerated atherosclerosis and CV disease ${ }^{43,44}$. Moreover, our 
group has described different epidemiological associations between lymphopenia and other conditions related to endothelial dysfunction, such as thrombotic microangiopathy, thrombotic thrombocytopenic purpura, and posterior reversible encephalopathy syndrome, in patients with SLE ${ }^{45,46}$. Additionally, it has been described that young men with ED have subclinical endothelial dysfunction, even without $\mathrm{CV}$ comorbidities ${ }^{47}$. Therefore, ED could be considered an early clinical sign of increased CV risk, with endothelial dysfunction potentially playing a key role ${ }^{48}$.

Currently, there is no specific information regarding pharmacological therapies in men with SLE and ED. This should be studied to address their efficacy and safety in this population. However, lifestyle modifications (weight loss, an appropriate diet, and increased physical activity $)^{49}$ could be recommended for patients with $\mathrm{ED}$, not only to improve erectile function, but also to reduce $\mathrm{CV}$ risk. Also, a prompt urology referral could help to individualize each case and the therapeutic options ${ }^{50}$.

Our work has several limitations. First, it was a transversal study, which did not allow addressing whether there are changes according to disease activity and treatment throughout time. It was performed only in Latin Americans, so these findings may not apply to other populations. Also, there was no neuropsychological assessment at the time of the study, which could have helped to find other factors influencing erectile function. Prospective studies should be performed, with evaluations regarding health-related quality of life and disease perception, as well as functional studies of penile vasculature.

Men with SLE have a strikingly high prevalence of ED, regardless of their young age. Glucocorticoid use and lymphopenia, both of which may cause endothelial dysfunction and lead to vascular damage, are independent novel risk factors for ED in these patients. Men with SLE are rarely asked about sexual function in their regular outpatient visits, even though most of them would agree to such an assessment by their rheumatologist.

\section{REFERENCES}

1. Tsokos GC. Systemic lupus erythematosus. N Engl J Med 2011;365:2110-21.

2. Murphy G, Isenberg D. Effect of gender on clinical presentation in systemic lupus erythematosus. Rheumatology 2013;52:2108-15.

3. Faezi ST, Hosseini Almodarresi M, Akbarian M, Gharibdoost F, Akhlaghi M, Jamshidi A, et al. Clinical and immunological pattern of systemic lupus erythematosus in men in a cohort of 2355 patients. Int J Rheum Dis 2014;17:394-9.

4. Boodhoo KD, Liu S, Zuo X. Impact of sex disparities on the clinical manifestations in patients with systemic lupus erythematosus: A systematic review and meta-analysis. Medicine 2016;95:e4272.

5. Garcia MA, Marcos JC, Marcos AI, Pons-Estel BA, Wojdyla D, Arturi A, et al. Male systemic lupus erythematosus in a Latin-American inception cohort of 1214 patients. Lupus 2005; 14:938-46.

6. Makhlouf A, Kparker A, Niederberger CS. Depression and erectile dysfunction. Urol Clin North Am 2007;34:565-74, vii.
7. Fan D, Liu L, Ding N, Liu S, Hu Y, Cai G, et al. Male sexual dysfunction and ankylosing spondylitis: A systematic review and metaanalysis. J Rheumatol 2015;42:252-7.

8. Cakar E, Dincer U, Kiralp MZ, Taskaynatan MA, Yasar E, Bayman EO, et al. Sexual problems in male ankylosing spondylitis patients: Relationship with functionality, disease activity, quality of life, and emotional status. Clin Rheumatol 2007;26:1607-13.

9. Lopes Gallinaro A, Silva CA, Rabelo CN Jr., Correia Caleiro MT, de Carvalho JF. Moderate/severe erectile dysfunction in patients with antiphospholipid syndrome. Lupus 2012;21:319-23.

10. Tristano AG. The impact of rheumatic diseases on sexual function. Rheumatol Int 2009;29:853-60.

11. Proietti M, Aversa A, Letizia C, Rossi C, Menghi G, Bruzziches R, et al. Erectile dysfunction in systemic sclerosis: effects of longterm inhibition of phosphodiesterase type-5 on erectile function and plasma endothelin-1 levels. J Rheumatol 2007;34:1712-7.

12. Hong P, Pope JE, Ouimet JM, Rullan E, Seibold JR. Erectile dysfunction associated with scleroderma: a case-control study of men with scleroderma and rheumatoid arthritis. J Rheumatol 2004;31:508-13.

13. Liu YF, Wen CY, Tu SH. On the relationship of male sexual dysfunction and ankylosing spondylitis. J Rheumatol 2015;42:2513.

14. Kumar R, Biggart JD, McEvoy J, McGeown MG. Cyclophosphamide and reproductive function. Lancet 1972; 1:1212-4.

15. Arnaud L, Nordin A, Lundholm H, Svenungsson E, Hellbacher E, Wikner J, et al. Effect of corticosteroids and cyclophosphamide on sex hormone profiles in male patients with systemic lupus erythematosus or systemic sclerosis. Arthritis Rheumatol 2017;69:1272-9.

16. Silva CA, Brunner HI. Gonadal functioning and preservation of reproductive fitness with juvenile systemic lupus erythematosus. Lupus 2007;16:593-9.

17. Silva CA, Hallak J, Pasqualotto FF, Barba MF, Saito MI, Kiss MH. Gonadal function in male adolescents and young males with juvenile onset systemic lupus erythematosus. J Rheumatol 2002;29:2000-5.

18. Ostensen $M$. New insights into sexual functioning and fertility in rheumatic diseases. Best Pract Res Clin Rheumatol 2004;18:219-32.

19. Vecchi AP, Borba EF, Bonfa E, Cocuzza M, Pieri P, Kim CA, et al. Penile anthropometry in systemic lupus erythematosus patients. Lupus 2011;20:512-8.

20. Impotence in systemic lupus erythematosus. J Rheumatol 1990;17:117-9.

21. Daleboudt GM, Broadbent E, McQueen F, Kaptein AA. The impact of illness perceptions on sexual functioning in patients with systemic lupus erythematosus. J Psychosom Res 2013;74:260-4.

22. Rabelo-Junior CN, Bonfa E, Carvalho JF, Cocuzza M, Saito O, Abdo $\mathrm{CH}$, et al. Penile alterations with severe sperm abnormalities in antiphospholipid syndrome associated with systemic lupus erythematosus. Clin Rheumatol 2013;32:109-13.

23. Lalani S, Pope J, de Leon F, Peschken C; Members of CaNIOS/1000 Faces of Lupus. Clinical features and prognosis of late-onset systemic lupus erythematosus: results from the 1000 Faces of Lupus study. J Rheumatol 2010;37:38-44.

24. Rosen RC, Cappelleri JC, Smith MD, Lipsky J, Pena BM. Development and evaluation of an abridged, 5-item version of the International Index of Erectile Function (IIEF-5) as a diagnostic tool for erectile dysfunction. Int J Impot Res 1999;11:319-26.

25. Morillo LE, Díaz J, Estevez E, Costa A, Méndez H, Dávila H, et al. Prevalence of erectile dysfunction in Colombia, Ecuador, and Venezuela: a population-based study (DENSA). Int J Impot Res 2002;14 Suppl 2:S10-8.

26. Bombardier C, Gladman DD, Urowitz MB, Caron D, Chang CH. Derivation of the SLEDAI. A disease activity index for lupus

Personal non-commercial use only. The Journal of Rheumatology Copyright (C) 2019. All rights reserved. 
patients. The Committee on Prognosis Studies in SLE. Arthritis Rheum 1992;35:630-40.

27. Gladman D, Ginzler E, Goldsmith C, Fortin P, Liang M, Urowitz M, et al. The development and initial validation of the Systemic Lupus International Collaborating Clinics/American College of Rheumatology damage index for systemic lupus erythematosus. Arthritis Rheum 1996;39:363-9.

28. Rosen RC, Fisher WA, Eardley I, Niederberger C, Nadel A, Sand M, et al. The multinational Men's Attitudes to Life Events and Sexuality (MALES) study: I. Prevalence of erectile dysfunction and related health concerns in the general population. Curr Med Res Opin 2004;20:607-17.

29. Giuliano FA, Leriche A, Jaudinot EO, de Gendre AS. Prevalence of erectile dysfunction among 7689 patients with diabetes or hypertension, or both. Urology 2004;64:1196-201.

30. Bacon CG, Mittleman MA, Kawachi I, Giovannucci E, Glasser DB, Rimm EB. A prospective study of risk factors for erectile dysfunction. J Urol 2006;176:217-21.

31. Grover SA, Lowensteyn I, Kaouache M, Marchand S, Coupal L, DeCarolis E, et al. The prevalence of erectile dysfunction in the primary care setting: importance of risk factors for diabetes and vascular disease. Arch Intern Med 2006;166:213-9.

32. Gerber RE, Vita JA, Ganz P, Wager CG, Araujo AB, Rosen RC, et al. Association of peripheral microvascular dysfunction and erectile dysfunction. J Urol 2015;193:612-7.

33. Chen SY, Choi CB, Li Q, Yeh WS, Lee YC, Kao AH, et al. Glucocorticoid use in patients with systemic lupus erythematosus: association between dose and health care utilization and costs. Arthritis Care Res 2015;67:1086-94.

34. Thomas A, Woodard C, Rovner ES, Wein AJ. Urologic complications of nonurologic medications. Urol Clin North Am 2003;30:123-31.

35. Uckert S, Fuhlenriede MH, Becker AJ, Stief CG, Scheller F, Knapp $\mathrm{WH}$, et al. Is there an inhibitory role of cortisol in the mechanism of male sexual arousal and penile erection? Urol Res 2003;31:402-6.

36. Braunstein GD. Endocrine causes of impotence. Optimistic outlook for restoration of potency. Postgrad Med 1983;74:207-17.

37. Buvat J, Maggi M, Gooren L, Guay AT, Kaufman J, Morgentaler A, et al. Endocrine aspects of male sexual dysfunctions. J Sex Med 2010;7:1627-56.

38. Doria A, Shoenfeld Y, Wu R, Gambari PF, Puato M, Ghirardello A, et al. Risk factors for subclinical atherosclerosis in a prospective cohort of patients with systemic lupus erythematosus. Ann Rheum Dis 2003;62:1071-7.
39. Souverein PC, Berard A, Van Staa TP, Cooper C, Egberts AC, Leufkens HG, et al. Use of oral glucocorticoids and risk of cardiovascular and cerebrovascular disease in a population based case-control study. Heart 2004;90:859-65

40. Salem S, Abdi S, Mehrsai A, Saboury B, Saraji A, Shokohideh V, et al. Erectile dysfunction severity as a risk predictor for coronary artery disease. J Sex Med 2009;6:3425-32.

41. Sanchez-Cruz JJ, Cabrera-Leon A, Martin-Morales A, Fernandez A, Burgos R, Rejas J. Male erectile dysfunction and health-related quality of life. Eur Urol 2003;44:245-53.

42. Jolly M. How does quality of life of patients with systemic lupus erythematosus compare with that of other common chronic illnesses? J Rheumatol 2005;32:1706-8.

43. Nunez J, Minana G, Bodi V, Nunez E, Sanchis J, Husser O, et al. Low lymphocyte count and cardiovascular diseases. Curr Med Chem 2011;18:3226-33.

44. Bhat T, Teli S, Rijal J, Bhat H, Raza M, Khoueiry G, et al. Neutrophil to lymphocyte ratio and cardiovascular diseases: A review. Expert Rev Cardiovasc Ther 2013;11:55-9.

45. Merayo-Chalico J, Apodaca E, Barrera-Vargas A, Alcocer-Varela J, Colunga-Pedraza I, Gonzalez-Patino A, et al. Clinical outcomes and risk factors for posterior reversible encephalopathy syndrome in systemic lupus erythematosus: a multicentric case-control study. J Neurol Neurosurg Psychiatry 2016;87:287-94.

46. Merayo-Chalico J, Demichelis-Gomez R, Rajme-Lopez S, Aparicio-Vera L, Barrera-Vargas A, Alcocer-Varela J, et al. Risk factors and clinical profile of thrombotic thrombocytopenic purpura in systemic lupus erythematosus patients. Is this a distinctive clinical entity in the thrombotic microangiopathy spectrum?: a case control study. Thromb Res 2014;134:1020-7.

47. Yao F, Huang Y, Zhang Y, Dong Y, Ma H, Deng C, et al. Subclinical endothelial dysfunction and low-grade inflammation play roles in the development of erectile dysfunction in young men with low risk of coronary heart disease. Int J Androl 2012;35:653-9.

48. Solomon H, Man JW, Jackson G. Erectile dysfunction and the cardiovascular patient: endothelial dysfunction is the common denominator. Heart 2003;89:251-3.

49. Gupta BP, Murad MH, Clifton MM, Prokop L, Nehra A, Kopecky SL. The effect of lifestyle modification and cardiovascular risk factor reduction on erectile dysfunction: a systematic review and meta-analysis. Arch Intern Med 2011;171:1797-803.

50. Hatzimouratidis K, Amar E, Eardley I, Giuliano F, Hatzichristou D, Montorsi F, et al. Guidelines on male sexual dysfunction: erectile dysfunction and premature ejaculation. Eur Urol 2010;57:804-14. 